\title{
Wahlen im Schatten der Flüchtlingskrise. Parteien, Wähler und Koalitionen im Umbruch
}

\author{
Fritz Plasser / Franz Sommer \\ Wien 2018 (= Schriftenreihe des Zentrums für Angewandte Politikforschung, Bd. 33): Facultas, 181 S.
}

Heinz P. Wassermann

FH JOANNEUM, Institut Journalismus und Public Relations, Austria

E-Mail: heinz.wassermann@fh-joanneum.at, Twitter: @heinzwassermann

Auch wenn die Wahlergebnisse zu österreichischen $\mathrm{Na}-$ tionalratswahlen Überraschungen bergen und Legislaturperioden von unterschiedlicher Dauer sind, so gibt es zwei wissenschaftliche Konstanten der Wahlanalysen: zum einen die vom Politikberater Thomas Hofer und der Journalistin Barbara Tóth herausgegebenen Sammelbände (vgl. Hofer/Tóth 2007, 2008, 2013, 2017), die vor allem ob der Kampagneninsights lesenswert sind. Zum anderen die von Fritz Plasser als Co-Autor bzw. als (Co-) Herausgeber (vgl. Müller/Plasser/Ulram (1995), Plasser (20IO), Plasser/Sommer/Ulram (I99I), Plasser/Ulram (1986, I987, I988, 2003, 2007), Plasser/Ulram/Ogris (Hg.) (1996), Plasser/Ulram/Sommer (Hg.) (2000)) publizierten, stark Empirie- und Umfrage-basierten Studien und/ oder Veröffentlichungen. Diese werden in Österreich im Gegensatz zur Bundesrepublik - von der öffentlichen Hand auffallend gering gefördert. Beide Ansätze sollten (!) für den einschlägig Interessierten eine unverzichtbare Lektüre sein.

„Die Flüchtlings- und Asylfrage hat zu einer politisch-kulturellen Spaltung der österreichischen Gesellschaft geführt, die zwischen wie innerhalb der Parteiwählerschaften zu konfliktären Auseinandersetzungen führt. In Verbindung mit der akuten politischen Vertrauenskrise, der verbreiteten Skepsis gegenüber der EU und der Polarisierung der Öffentlichkeit in Flüchtlings- und Asylfragen befindet sich die politische Kultur Österreichs in einer angespannten Bewährungsprobe, deren Ausgang aus heutiger Sicht noch nicht absehbar ist" (Plasser/Seeber 2017, 360). Das las sich in der im Wahljahr 2017 publizierten Zweitauflage des Sammelbandes „Die österreichische Demokratie im internationalen Vergleich“ wie ein Menetekel. Das inhaltlich vermisch- te Asyl-, Flucht- und Migrationsthema war neben dem „Wunsch nach Erneuerung, Veränderung und Überwindung des politischen Stillstands“ und dem „hohe[n] Stellenwert koalitionstaktischer Erwägungen“ einer der „drei Faktoren“, die „den Ausgang dieser spektakulären Wahl entscheidend beeinflusst" (I49) hat.

Diese auf den ersten Blick recht simple Formel wird argumentativ doppelt fundiert. Im ersten Teil (I3-50) des Buches spannen die Autoren weite historisch-thematische Bögen: Die Erosion der Parteibindungen, das damit verbundene, massiv veränderte Wahlverhalten und der geradezu kollabierende Imageverlust der nur noch „mittelgroßen Koalition“ (vgl. Khol 20I4) fungieren als Struktur bzw. Basis für die eruptiven politischen Veränderungen des I5. Oktober 20I7. Als unmittelbar relevant für das Wahlergebnis führen Plasser und Sommer neben der Flüchtlingskrise 2015 den damit einhergehenden Vertrauensverlust in die politischen Akteure auf nationaler wie auch auf internationaler Ebene an. Er wirkte sich sowohl auf die Landtagswahlen in Oberösterreich und Wien als auch auf die Bundespräsidentschaftswahl(en) aus. Hinzu kam der „Kurz-Effekt“ (7I): „In Summe beziehen sich zwei Drittel der zentralen ÖVP-Wahlmotive auf die Persönlichkeit des ÖVP-Kanzlerkandidaten." (IOO) Dies einerseits durch das mit der Übernahme der Obmannschaft sowie die Aufkündigung der Koalition gezeigte Leadership des „, absoluten Jahrhundert-Talent[s]“" (Kurier 20I7) Sebastian Kurz sowie andererseits durch die Repositionierung der Volkspartei in der Migrations- bzw. Integrationsfrage. Sie konnte dadurch „in Kompetenzbereiche vordringen, die bislang weitgehend von der FPÖ besetzt waren“ (87), was den „,Markenkern““ (9I) der in diesem 
Fall tatsächlich neuen Volkspartei beträchtlich erweiterte.

Plasser und Sommer rühren gewissermaßen einen datenbasierten Cocktail aus a) lang- und längerfristigen Entwicklungen und b) punktuellen Ereignissen seit 2015 an, die summa summarum wie ein Katalysator zugunsten des Wahlerfolgs der Volkspartei wirkten. Somit - aber das ist für Plasser-Publikationen typisch und macht sie im Bereich der Wahlforschung unverzichtbar - wird jeder, der sich durch die Datenreihen, Daten$s$ (ch)ätze, Tabellen und Grafiken müht (und gelegentlich ist der Text alles andere als leicht lesbar, was für PlasserPublikationen auch nicht neu ist), nach der Lektüre reich belohnt. Bedauernswert ist, dass - im Gegensatz zu den ungebrochen produktiven Reihen „Studien zu Politik und Verwaltung" oder der "Schriftenreihe des Forschungszentrums für politisch-historische Studien der Dr.-Wilfried-Haslauer-Bibliothek, Salzburg" - die Publikationsdichte des „Zentrums für Angewandte Politikforschung" im letzten Jahrzehnt beinahe zum Erliegen gekommen ist.

Abschließend ist $\mathrm{zu}$ bemerken, dass es, angesichts von 640.00o ÖVP-Zuwanderern (vgl. SORA 20I7), aus Sicht der Volkspartei durchaus ein „außerordentliches Wahlergebnis“ (7) war. Allerdings reichte es nicht an den "Jahrhundertstrom" (Hofinger et. al. 2003 I59) bzw. die „Erdrutschwahlen“ (Plasser et. al. 2003 97) oder das „Erdbeben“ (ebda II5) von 2002 mit 890.000 ÖVP-Zuwanderern (vgl. SORA 2002) heran. Es zeigt sich aber insofern eine Parallele, als es der Volkspartei 20I7, wie I5 Jahre zuvor, gelang, in verloren gegangene bzw. neue Wählersegmente einzudringen. Allerdings verlor sie diese spätestens ab bzw. mit der Nationalratswahl 2006 sukzessive wieder. 2003 wiesen Plasser, Ulram und Seeber zwar kassandramäßig darauf hin, dass „die Wählerschaft der ÖVP (...) eine deutlich abgeschwächte Bindung an die Partei“ (Plasser et. al. 2003 III) aufweist. Diese „,Analyse (...) wurde registriert, aufgrund der starken Fokussierung auf die Regierungsarbeit wurde jedoch die Parteiarbeit zweitrangig gesehen und eine Strategie zur Wählerbindung der mehr als 800 ooo neu gewonnenen Wähler verabsäumt.“' (Wassermann 20I3, I72, Anm. 315)

\section{Literatur}

Hofer, Thomas/Barbara Tóth (Hg.) (2007), Wahl 2006. Kanzler, Kampagnen, Kapriolen. Analysen zur Nationalratswahl, Wien/Berlin: LIT.

Hofer, Thomas/Barbara Tóth (Hg.) (2008), Wahl 2008. Strategien - Sieger - Sensationen, Wien: Molden.

Hofer, Thomas/Barbara Tóth (Hg.) (2O13), Wahl 2013. Macht, Medien, Milliardäre, Wien/Berlin: LIT.

Hofer, Thomas/Barbara Tóth (Hg.) (2017), Wahl 2017. Loser, Leaks \& Leadership, Wien: ÄrzteVerlag.
Hofinger, Christoph/Günther Ogris/Eva Thalhammer (2003), Der Jahrhundertstrom: Wahlkampfverlauf, Wahlmotive und Wählerströme im Kontext der $\mathrm{Na}-$ tionalratswahl 2002, in: Plasser, Fritz/Peter A. Ulram (Hg.), Wahlverhalten in Bewegung. Analysen zur Nationalratswahl 2002, Wien: WUV-Univ.-Verl. (= Schriftenreihe des Zentrums für Angewandte Politikforschung, Bd. 28), 159-190.

Khol, Andreas (20I4), MIGROKO vor dem Ende? Auf dem Weg in eine neue Republik, in: Khol, Andreas/ Günther Ofner/Stefan Karner/Dietmar Halper $(\mathrm{Hg})$, Österreichisches Jahrbuch für Politik 20I3, Wien/ Köln/Weimar: Böhlau, 3-I4.

Kurier (2OI7), ÖVP-Führungsdebatte: Appelle an Mitterlehner und Kurz, in: kurier.at, 07. OI. 20I7, Internet: https://kurier.at/politik/inland/oevp-fuehrungsdebatte-appelle-an-mitterlehner-und-kurz/239.799.8II (Zugriff: 28. 06. 2019).

Müller, Wolfgang C./Fritz Plasser/Peter A. Ulram. (Hg.) (1995), Wählerverhalten und Parteienwettbewerb. Analysen zur Nationalratswahl I994, Wien: Signum (= Schriftenreihe des Zentrums für Angewandte Politikforschung, Bd. 8).

Plasser, Fritz (Hg.) (2010), Politik in der Medienarena. Praxis politischer Kommunikation in Österreich, Wien: Facultas (= Schriftenreihe des Zentrums für Angewandte Politikforschung, Bd. 3I).

Plasser, Fritz/Gilig Seeber (2017), Politische Kultur und Demokratiebewusstsein in der Zweiten Republik im internationalen Vergleich, in: Helms, Ludger/David M. Wineroither (Hg), Die österreichische Demokratie im internationalen Vergleich, Baden-Baden: Nomos (= Politik und Demokratie in den kleinen Ländern Europas, Bd. I), 2. überarbeitete und aktualisierte Auflage, 337-364.

Plasser, Fritz/Franz Sommer/Peter A. Ulram (199I), Eine Kanzler- und Protestwahl. Wählerverhalten und Wahlmotive bei der Nationalratswahl 1990, in: Kohl, Andreas/Günther Ofner/Alfred Stirnemann (Hg.). Österreichisches Jahrbuch für Politik 199o, Wien/München: Verlag für Geschichte und Politik/R. Oldenburg Verlag, 3I-80.

Plasser, Fritz/Peter A. Ulram (1986), Ein Beben mit Folgen. Die Präsidentschaftswahl 1986, in: Österreichische Monatshefte, 42(4), 6-IO.

Plasser, Fritz/Peter A. Ulram (1987), Das Jahr der Wechselwähler. Wahlen und Neustrukturierung des österreichischen Parteiensystems I986, in: Kohl, Andreas/ Günther Ofner/Alfred Stirnemann (Hg.), Österreichisches Jahrbuch für Politik I986, Wien/München: Verlag für Geschichte und Politik/R. Oldenburg Verlag, 95-I49.

Plasser, Fritz/Peter A. Ulram (1988), Großparteien in der Defensive. Die österreichische Parteien- und Wählerschaft nach der Nationalratswahl 1986, in: Pelinka, 
Anton/Fritz Plasser (Hg.), Das österreichische Parteiensystem, Wien/Köln/Graz: Böhlau (= Studien zu Politik und Verwaltung, Bd. 22), 79-IO2.

Plasser, Fritz/Peter A. Ulram (Hg.) (2003), Wahlverhalten in Bewegung. Analysen zur Nationalratswahl 2002, Wien: WUV-Univ.-Verl. (= Schriftenreihe des Zentrums für Angewandte Politikforschung, Bd. 28).

Plasser, Fritz/Peter A. Ulram (Hg.) (2007), Wechselwahlen. Analysen zur Nationalratswahl 2006, Wien: Facultas (= Schriftenreihe des Zentrums für Angewandte Politikforschung, Bd. 30).

Plasser, Fritz/Peter A. Ulram/ Günther Ogris (Hg.) (1996), Wahlkampf und Wählerentscheidung. Analysen zur Nationalratswahl 1995, Wien: Signum (= Schriftenreihe des Zentrums für Angewandte Politikforschung, Bd. II).

Plasser, Fritz/Peter A. Ulram/Gilg Seeber (2003), Erdrutschwahlen: Momentum, Motive und neue Muster im Wahlverhalten, in: Plasser, Fritz/Ulram, Peter A. (Hg.), Wahlverhalten in Bewegung. Analysen zur Nationalratswahl 2002, Wien: WUV-Univ.-Verl. (= Schriftenreihe des Zentrums für Angewandte Politikforschung, Bd. 28), 97-I57.

Plasser, Fritz/Peter A. Ulram/ Franz Sommer (Hg.) (2000), Das österreichische Wahlverhalten, Wien: Signum (= Schriftenreihe des Zentrums für Angewandte Politikforschung, Bd. 2I).

SORA (2002), Nationalratswahl 2002, Internet: https:// www.sora.at/themen/wahlverhalten/wahlanalysen/ nrwo2.html (Zugriff: 28. 07. 2018).

SORA (2017), Nationalratswahl 20I7: Wählerstromanalyse, Internet: https://www.sora.at/fileadmin/downloads/wahlen/20I7_NRW-Waehlerstroeme_Tabellen.pdf (Zugriff: 28. 07. 2018).

Wassermann, Heinz P. (2013), „Es ist ein täglicher Wettkampf um die Schlagzeile - oder einen Punkt beim Wähler". Regierungs- und Parteikommunikation 1999/2000 bis 2006, in: Kriechbaumer, Robert/Franz Schausberger (Hg.), Die umstrittene Wende. Österreich 2000-2006, Wien/Köln/Weimar: Böhlau (= Schriftenreihe des Forschungsinstitutes für politisch-historische Studien der Dr.-Wilfried-HaslauerBibliothek, Bd. 43), I23-182. 
